\title{
Acoustophoresis of hollow and core-shell particles in two- dimensional resonance modes
}

\author{
Journal Article \\ Author(s): \\ Leibacher, Ivo; Dietze, Wolfgang; Hahn, Philipp; Wang, Jingtao; Schmitt, Steven; Dual, Jürg \\ Publication date: \\ 2014-03 \\ Permanent link: \\ https://doi.org/10.3929/ethz-b-000077484 \\ Rights / license: \\ In Copyright - Non-Commercial Use Permitted \\ Originally published in: \\ Microfluidics and Nanofluidics 16(3), https://doi.org/10.1007/s10404-013-1240-7
}




\title{
Acoustophoresis of hollow and core-shell particles in two-dimensional resonance modes
}

\author{
Ivo Leibacher • Wolfgang Dietze • Philipp Hahn • \\ Jingtao Wang $\cdot$ Steven Schmitt $\cdot$ Jürg Dual
}

Received: 8 March 2013/Accepted: 20 July 2013/Published online: 7 August 2013

(C) Springer-Verlag Berlin Heidelberg 2013

\begin{abstract}
Motivated by the applications of ultrasonic particle manipulation in a biotechnological context, a study on acoustophoresis of hollow and core-shell particles is presented with analytical derivations, numerical simulations and confirming experiments. For a long-wavelength calculation of the acoustic radiation forces, the Gor'kov potential of hollow, air-filled particles and particles with solid or fluid core and shell is derived. The validity as well as the applicable range of the long-wavelength calculation is evaluated with numerical simulations in Comsol Multiphysics ${ }^{\circledR}$. The results are experimentally verified in the acoustic field of an intrinsically two-dimensional fluid resonance mode, which allows for a more complex analysis than the common one-dimensional ultrasonic standing waves or their superposition to two-dimensional fields. Experiments were conducted with hollow glass particles $(13.9 \mu \mathrm{m}$ diameter $)$ in a microfluidic chamber of $1.2 \mathrm{~mm} \times 1.2 \mathrm{~mm} \times 0.2 \mathrm{~mm}$ on a silicon-based device with piezoelectric excitation around $870 \mathrm{kHz}$. The described resonance mode is of additional interest for particle trapping and medium exchange on certain particle types,
\end{abstract}

Electronic supplementary material The online version of this article (doi:10.1007/s10404-013-1240-7) contains supplementary material, which is available to authorized users.

I. Leibacher $(\varangle) \cdot$ W. Dietze $\cdot$ P. Hahn · J. Wang · J. Dual Department of Mechanical and Process Engineering, Institute of Mechanical Systems (IMES), Swiss Federal Institute of Technology (ETH Zurich), Tannenstrasse 3, 8092 Zurich, Switzerland

e-mail: leibacher@imes.mavt.ethz.ch

\section{S. Schmitt}

Department of Biosystems Science and Engineering, Bioprocess Laboratory (BPL), Swiss Federal Institute of Technology (ETH Zurich), Mattenstrasse 26, 4058 Basel, Switzerland and it reveals a novel approach for particle characterization or separation.

Keywords Acoustophoresis - Acoustofluidics · Ultrasonic particle manipulation · Core-shell particles · Gor'kov potential

\section{Introduction}

The movement of particles by the forces of an acoustic field, namely acoustophoresis, continues to hold significant promise for emerging applications in bio- and microtechnology on lab-on-a-chip systems. Ultrasonic particle manipulation offers methods for microfluidic tasks such as the handling (Manneberg et al. 2009), positioning (Oberti et al. 2007), separation (Laurell et al. 2007) and characterization (Hartono et al. 2011) of cell-sized particles, as recently reviewed in Lab on a Chip (Bruus et al. 2011).

The main focus of recent works on acoustophoresis has been on the manipulation of homogeneous, full particles. However, hollow and filled core-shell microparticles, double emulsions as well as droplets with encapsulated microbeads have experienced an increased interest in the microfluidic community. Such particles exhibit properties that are substantially different from those of homogeneous particles, thus making them attractive from both a scientific and a technological viewpoint. Microfluidic platforms for the fabrication of such particles are readily available: Utada et al. (2005) proposed the generation of double emulsions on a microcapillary device. Hennequin et al. (2009) and Choi et al. (2009) synthesized microcapsules with controlled geometrical and mechanical properties. Jeong et al. (2012) recently reported on double emulsion droplets, silica capsules and microfluidic emulsification. A variety of 
biotechnological applications including encapsulation and delivery of drugs, cells, microbeads and nutrients, high throughput screening, catalysis, separation, sensors and microreactors are feasible (Griffiths and Tawfik 2006), whereas intrinsic advantages of microparticles come into play, for example their large specific surface area and tunable morphologies. Furthermore, ultrasound contrast agents as well as commercially available magnetic particles are based on a core-shell structure. Acoustophoresis offers a valuable manipulation method for these particles, which is one of the motivations of the paper at hand. In addition, hollow particles allow their buoyancy to be tuned to the suspending fluid, such that acoustophoresis processes could be further optimized with respect to minimal particle sedimentation.

Acoustic theory on hollow and shelled/filled particles in nonviscous fluids has been well studied over the past few decades. Hasegawa et al. (1993) derived the acoustic radiation pressure on a spherical shell placed in a plane progressive wave. Mitri (2005) extended Hasegawa's work by deriving the acoustic radiation force on elastic and viscoelastic shells in standing wave fields. Their derivation is valid for arbitrary ratios between particle size and wavelength. As distinguished from this work, regarding acoustophoretic theory, the goal of this paper lies in the calculation of acoustic radiation forces for hollow and core-shell particles in the framework of Gor'kov's theory (Gor'kov 1962) in order to provide an expedient longwavelength approximation. This approach based on Gor'kov's theory results in a simplification of the equations, so they can efficiently be evaluated with analytical calculations and not only by complex and numerically intensive calculations on computers. Furthermore, the derived equations give insight into the underlying physical effects. The validity of our approach is analyzed with numerical studies, which confirmed our approach in the typical wavelength range of applied acoustophoresis.

To provide a complete picture, enlightening experiments were conducted in a rectangular microfluidic chamber with a similar design as Manneberg et al. (2008b), but in a different operation mode. Whereas experimental acoustophoresis on micro-electro-mechanical systems (MEMS) dealt mostly with one-dimensional ultrasonic standing waves or their superposition to two-dimensional fields so far (Oberti et al. 2007; Manneberg et al. 2008b), here, we employ a different and intrinsically two-dimensional resonance mode. This resonance mode within a rectangular chamber geometry was chosen because it reveals particledependent acoustophoretic effects which are not observable in one-dimensional standing waves within common channel geometries or in the superposition of such onedimensional fields within chambers. Additional to the experimental evaluations of this novel resonance mode itself, it allows to confirm our coinciding analytic and numerical findings regarding the specific acoustophoretic behavior of hollow particles. A discussion of the varying force potentials within the chamber is a further step toward particle-dependent acoustophoresis with its promise for particle characterization and separation. The chamber will also be discussed in the context of its biotechnological applications, namely acoustic particle traps (Evander and Nilsson 2012) for long-term microscopy studies (Vanherberghen et al. 2010), analysis of perfused particle clusters (Lilliehorn et al. 2005) with medium exchange (Evander et al. 2007), bead-based bioaffinity assays (Wiklund et al. 2012; Wiklund and Hertz 2006) and the selective trapping of bioparticles (Svennebring et al. 2009).

The studies of this paper will allow to expand acoustophoresis on hollow and core-shell particles. We aim at providing a theoretical and experimental basis for the onchip handling of such microparticles, paving the way for their manifold biotechnological applications. Along this way, novel particle-dependent aspects of two-dimensional ultrasonic particle manipulation are revealed.

\section{Acoustophoretic theory}

\subsection{Basic equations}

The basic equations for the calculation of acoustophoretic forces on particles are recapitulated in the following. The Gor'kov potential $U$ (Gor'kov 1962; Bruus 2012b) in the acoustic domain reads:

$U=2 \pi r_{o}^{3} \rho_{w a}\left(\frac{\left\langle p_{1}^{2}\right\rangle}{3 \rho_{w a}^{2} c_{w a}^{2}} f_{1}-\frac{\left\langle v_{1}^{2}\right\rangle}{2} f_{2}\right)$

with the outer particle radius $r_{o}$, the density $\rho_{w a}$ and the speed of sound $c_{w a}$ in the fluid (here water), the first-order pressure and velocity fields $p_{1}, v_{1}$ and the factors $f_{1}, f_{2}$ which depend on the particle and fluid materials. $\rho_{w a}$ is the density of water in the quiescent state (namely the zero order density $\left.\rho_{0}\right) . v_{1}$ describes the magnitude of the real part of the complex velocity field vector, $v_{1}=\left\|\operatorname{Re}\left(\mathbf{v}_{1}\right)\right\|_{2}$. Time averaging is denoted by $\langle$.$\rangle . The Gor'kov potential is$ valid for particles with $r_{o}$ « $\lambda$ with the acoustic wavelength $\lambda$, in other words, in the long-wavelength range. Particles in the acoustic domain are attracted to the minimum of the Gor'kov potential $U$, which is a particle-dependent weighted sum of $\left\langle p_{1}^{2}\right\rangle$ and $\left\langle v_{1}^{2}\right\rangle$. The acoustic radiation force $\mathbf{F}$ on a particle equals

$\mathbf{F}=-\nabla U$

The first factor $f_{1}$ yields

$f_{1}=1-\frac{\kappa_{p}}{\kappa_{w a}}$ 
with the compressibility $\kappa_{p}$ of the particle material and $\kappa_{w a}$ of the surrounding water. Hereby, the compressibility $\kappa$ is defined as

$\kappa=-\frac{1}{V} \frac{\partial V}{\partial p}$

with the volume $V$. For fluids, the compressibility is calculated as

$\kappa=\frac{1}{\rho c^{2}}$

For a solid, its compressibility is the inverse of the bulk modulus $K$ of elasticity:

$\kappa=\frac{1}{K}=\frac{3(1-2 v)}{E}$

with Young's modulus $E$ and Poisson's ratio $v$. The second factor $f_{2}$ determines the influence of the velocity field in the Gor'kov potential. With the particle density $\rho_{p}$ it is given as

$f_{2}=\frac{2\left(\rho_{p}-\rho_{w a}\right)}{2 \rho_{p}+\rho_{w a}}$.

\subsection{Derivation of $f_{1}, f_{2}$ for hollow particles}

We propose the calculation of acoustic radiation forces on hollow particles, i.e., particles with a linear elastic solid shell filled with air, by deriving their factors $f_{1}, f_{2}$ in the Gor'kov potential (Eq. 1). This approach is outlined in the following by density and compressibility considerations. The derivation of these factors is motivated by their influence on the force potentials of later experiments, where they caused a particle-dependent acoustophoretic response.

For the derivations of the next two sections, we build on certain assumptions: the long-wavelength assumption and spherically symmetric particle deformation, both underlying Gor'kov's theory, and the assumption of linear elasticity. The first two limitations will be clarified with numerical simulations in Sect. 2.4. Concerning the third limitation, a linear analysis is appropriate for the particles discussed here; however, a nonlinear analysis might become necessary for shells undergoing large deformations.

For the calculation of $f_{2}$ from Eq. 7, the spatially averaged density $\bar{\rho}_{p}$ of a hollow particle is relevant. It is calculated by volume considerations as

$\bar{\rho}_{p}=\rho_{\text {shell }}\left(1-\alpha^{3}\right)$

with the density $\rho_{\text {shell }}$ of the shell's bulk material and the ratio $\alpha=r_{i} / r_{o}$ between the inner particle radius $r_{i}$ and the outer radius $r_{o}$ as illustrated in Fig. 1, while the density of the air fill is neglected.
Fig. 1 Illustration of a hollow glass particle (LSM image, Kisker $P B G H-18$ ) with outer radius $r_{o} \approx 7 \mu \mathrm{m}$, inner radius $r_{i}$ and outer and inner pressures $p_{o}, p_{i}$ hollow particle has to be found. Its derivation in linear elasticity is based on three cornerstones: The equilibrium equations, strain-displacement relations and pressure boundary conditions. Spherical coordinates are particularly suited for this case. Based on these equations, for a pressurized hollow sphere the spherically symmetric displacement $\mathbf{u}$ at a position $r \mathbf{e}_{r}$ (where $\mathbf{e}_{r}$ is a unit vector in radial direction) can be derived as outlined by, e.g., Bower (2009). The result is:

$$
\begin{aligned}
\mathbf{u}(r)= & \frac{1}{2 E\left(r_{o}^{3}-r_{i}^{3}\right) r^{2}}\left\{2\left(p_{i} r_{i}^{3}-p_{o} r_{o}^{3}\right)[1-2 v] r^{3}\right. \\
& \left.+\left(p_{i}-p_{o}\right)[1+v] r_{o}^{3} r_{i}^{3}\right\} \mathbf{e}_{r}
\end{aligned}
$$

with outer and inner pressures $p_{o}$ and $p_{i}$ and the material parameters $E, v$ of the shell material. In our case, the outer pressure equals the atmospheric pressure $p_{\text {atm }}$ (zero order) plus the first-order pressure perturbation $p_{1}$ of the acoustic field, $p_{o}=p_{a t m}+p_{1}$. The inner pressure is assumed to be approximately constant at $p_{i}=p_{\text {atm }}$ due to a filling gas, e.g., air.

Then, the overall volume $V$ of the deformed particle is

$V=\frac{4 \pi\left(r_{o}+\mathbf{u}\left(r_{o}\right) \cdot \mathbf{e}_{r}\right)^{3}}{3}$

Now the compressibility $\kappa_{p}$ of the particle around an initial equilibrium state with $p_{o}=p_{i}=p_{a t m}$ can be derived. Assuming $p_{\text {atm }}=0$, a small deviation from the initial state is neglected, which is valid as $\left|\mathbf{u}_{\text {initial }}\left(r_{o}\right)\right|<<r_{o}$ for typical solid materials at ambient pressure. With Eq. 4, it follows that:

$\kappa_{p}=\frac{3}{2 E\left(1-\alpha^{3}\right)}\left(2-4 v+\alpha^{3}[1+v]\right)$

As a plausibility check, it can be seen that with $r_{i}=0$, the above equation equals the bulk compressibility of a homogeneous particle, Eq. 6.

With Eqs. 3 and 7, we plotted the values of $f_{1}$ and $f_{2}$ in Fig. 2. For hollow particles with small values of $\alpha, f_{2}$ is similar compared to the case of a homogeneous particle of the same material which would be at $\alpha=r_{i} / r_{o}=0$. However, as $\alpha$ increases beyond 0.5 , we can observe how $f_{2}$ varies substantially-even the sign of $f_{2}$ can easily be changed as for the hollow particles which will be evaluated 
in the experimental Sect. 3 (hollow glass particles Kisker $P B G H-18$, material parameters given in Table 1). On the other hand, $f_{1}$ is found to be less sensitive to the inner radius of a hollow particle. A common value to characterize the particle behavior in a one-dimensional standing wave is the acoustophoretic contrast factor (Yosioka and Kawasima 1955):

$\phi=\frac{f_{1}}{3}+\frac{f_{2}}{2}=\frac{1}{3}\left(\frac{5 \frac{\rho_{p}}{\rho_{w a}}-2}{2 \frac{\rho_{p}}{\rho_{w a}}+1}-\frac{\kappa_{p}}{\kappa_{w a}}\right)$

Most common particles (e.g., glass, polystyrene, cells) exhibit $\phi>0$, so they are attracted to the pressure nodes of a one-dimensional standing wave. Particles with $\phi<0$ [e.g., lipid particles, air bubbles smaller than their resonant size (Blake 1949)] are moved toward the pressure antinodes. In Fig. 2, it becomes clear that-depending on their hollowness-hollow solid particles with $\phi<0$ can be fabricated even from a material which yields $\phi>0$ for homogeneous particles. The relevance of this interesting finding for biotechnological separations will be discussed in the conclusions.

\subsection{Derivation of $f_{1}, f_{2}$ for core-shell particles}

Whereas the last section covered hollow particles with negligible core material properties, here we will proceed to particles with a solid or fluid core and shell material. Figure 3 gives a sketch of such a particle.

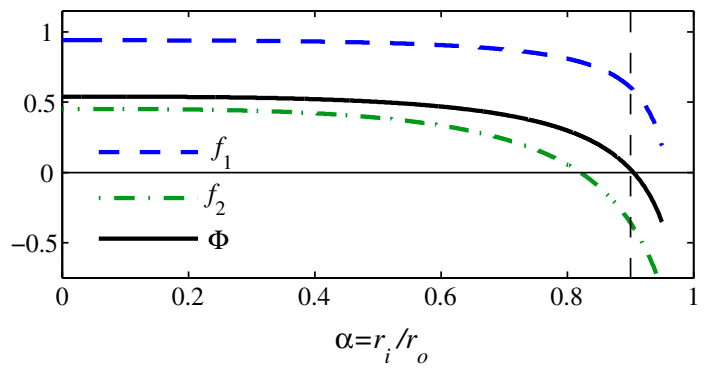

Fig. 2 The prefactors $f_{1}$ and $f_{2}$ of Gor'kov's potential for a hollow glass particle and its acoustophoretic contrast factor $\phi$ are plotted over the ratio $\alpha$ between its inner and outer radius $r_{i}$ and $r_{o}$. The vertical line denotes this ratio for the hollow glass particles of the later experiments
For the calculation of $f_{2}$, the spatially averaged density of the particle yields

$\bar{\rho}_{p}=\rho_{\text {shell }}\left(1-\alpha^{3}\right)+\rho_{\text {core }} \alpha^{3}$

To determine $f_{1}$, as in the last section, we calculate a particle's overall compressibility $\kappa_{p}$. First, the compressibility $\kappa_{i}$ of the fluid or solid core material follows from Eqs. 5 or 6. According to Eq. 4, we can then write

$\frac{\partial p_{i}}{\partial u_{i}}=-\frac{1}{V \kappa_{i}} \frac{\partial V}{\partial u_{i}}=\frac{-S}{V \kappa_{i}}=\frac{-3}{r_{i} \kappa_{i}}$

with $u_{i}=\mathbf{u}\left(r_{i}\right) \cdot \mathbf{e}_{r}$ and the particle surface area $S$, so for small volume variations, the pressure $p_{i}$ can be linearized as

$p_{i}=\frac{-3 u_{i}}{r_{i} \kappa_{i}}+\mathcal{O}\left(u_{i}^{2}\right)$

where we neglect the $\mathcal{O}\left(u_{i}^{2}\right)$ terms.

Now, we have to distinguish between solid and fluid shell materials. First, we will treat particles with a solid shell, then particles with a fluid shell. For a linear elastic solid shell of material parameters $E$ and $v$, with Eqs. 15 and 9 evaluated at $r_{o}$ and $r_{i}$, we can calculate the compressibility of the overall particle with an analogous calculation to that in the last section:

$$
\begin{aligned}
\kappa_{p}= & \left\{-3(1+v)\left(-3+E \kappa_{i}+6 v\right) \alpha^{3}\right. \\
+ & \left.3(-1+2 v)\left(3+2 E \kappa_{i}+3 v\right)\right\} \\
& \cdot\left\{2 E\left(-3+E \kappa_{i}+6 v\right) \alpha^{3}-E\left(3+2 E \kappa_{i}+3 v\right)\right\}^{-1}
\end{aligned}
$$

A first special case is when $\kappa_{i} \rightarrow \infty$, which means an approximation for gases such as air. Then, the above equation simplifies into the same result as for the hollow particles, Eq. 11. Further, special cases are plausible: With $r_{i}=r_{o}$, the equation yields $\kappa_{p}=\kappa_{i}$. With $r_{i}=0$, we get again the bulk compressibility of a homogeneous particle, Eq. 6, and if the compressibility of core and shell is equal, the dependency on $\alpha$ cancels out.

In Fig. 4, the above equation is plotted for the example of a glass-shelled particle. Indeed, the calculation with an air core results in almost the same curve as for the simpler approximation of a hollow particle, Eq. 11. A fill with

Table 1 Material parameters and particle properties at room temperature. $f_{1}$ and $f_{2}$ are calculated for particles in water as suspending fluid

\begin{tabular}{llllll}
\hline Material & Speed of sound $(\mathrm{m} / \mathrm{s})$ & Density & $f_{1}$ & $f_{2}$ & Diameter/other \\
\hline Water (Bruus 2012a) & $c_{w a}=1497$ & $\rho_{w a}=998 \mathrm{~kg} / \mathrm{m}^{3}$ & 0 & 0 & \\
Copolymer (Oberti et al. 2007) & $c_{c o}=3000$ & $\rho_{c o}=1050 \mathrm{~kg} / \mathrm{m}^{3}$ & 0.76 & 0.034 & $2 r_{o}=17 \mu \mathrm{m}$ \\
Ca-alginate 2.5 \% (Salsac et al. 2011) & $c_{a l}=1533$ & $\rho_{a l}=1096 \mathrm{~kg} / \mathrm{m}^{3}$ & 0.13 & 0.061 & $2 r_{o} \approx 100 \mu \mathrm{m}$ \\
Pyrex (Bruus 2012a) & $c_{p y}=5661$ & $\rho_{p y}=2230 \mathrm{~kg} / \mathrm{m}^{3}$ & 0.94 & 0.45 & $E=63 \mathrm{GPa}, v=0.22$ \\
Hollow glass (PBGH-18) & & $\bar{\rho}_{h o}=600 \mathrm{~kg} / \mathrm{m}^{3}$ & 0.602 & -0.362 & $2 r_{o} \approx 13.9 \mu \mathrm{m}, 2 r_{i} \approx 12.5 \mu \mathrm{m}$ \\
\hline
\end{tabular}




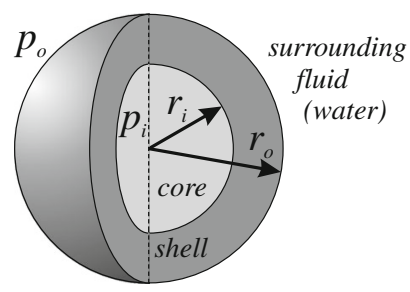

Fig. 3 Sketch of a filled spherical particle, consisting of a core and a shell, both either fluid or solid. $p_{o}, p_{i}$ are the outer and inner pressures

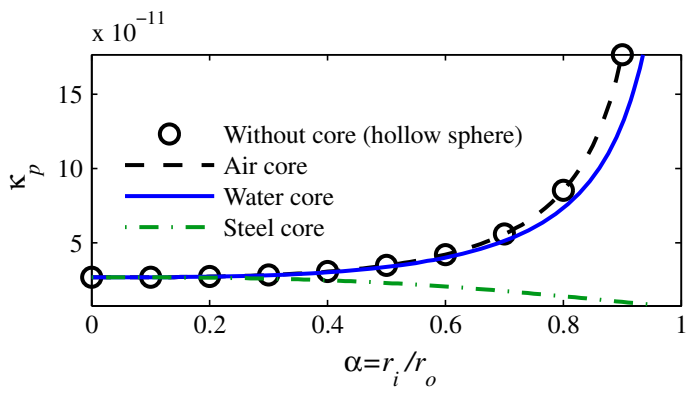

Fig. 4 Compressibility $\kappa_{p}$ of a glass-shelled particle for several core materials

water leads to a more compressible particle compared to a homogeneous glass particle because $\kappa_{\text {water }}>\kappa_{\text {glass }}$. Vice versa, a glass particle becomes less compressible when filled with steel because $\kappa_{\text {steel }}<\kappa_{\text {glass }}$.

Now we consider a particle with a fluid shell around a fluid core (double emulsion) or solid core. With Eq. 4 and $p_{o}=p_{i}$ in this case, we can derive the particle's compressibility

$\kappa_{p}=-\frac{1}{V} \frac{\partial\left(V_{i}+V_{o}\right)}{\partial p_{o}}=\frac{1}{V}\left(V_{i} \kappa_{i}+V_{o} \kappa_{o}\right)$

$=\kappa_{i} \alpha^{3}+\kappa_{o}\left(1-\alpha^{3}\right)$

with the volume $V_{i}$ of the core, the volume $V_{o}$ of the shell, the particle volume $V=V_{i}+V_{o}$ and the compressibility $\kappa_{o}$ of the fluid shell material. The result equates to the spatially averaged compressibility of the core-shell particle with the special cases $\kappa_{p}=\kappa_{i}$ for $r_{i}=r_{o}$ and $\kappa_{p}=\kappa_{o}$ for $r_{i}=0$ or for $\kappa_{o}=\kappa_{i}$.

With these compressibilities, $f_{1}$ can be calculated according to Eq. 3. The formulas also allow one to deal with particles having more than one shell layer by calculating the compressibility of the core and most inner shell first, reinserting this result in a next step as $\kappa_{i}$ seen by the next shell layer and so forth.

The discussed findings are of interest concerning acoustophoresis of biological cells. The approach to model a cell with a core-shell particle as derived above suggests itself. Lim et al. (2006) give a review on the various existing mechanical cell models, where it becomes clear that the modeling of a cell as a core-shell particle is a rather rough model, because it does not include viscoelasticity. Nevertheless, several researchers report evidence of an elastic nature to cells (Caille et al. 2002; Mishra et al. 2012), whereas the varying mechanical properties of the organelles contribute differently to an overall acoustic radiation force. Concluding, we believe our model to be useful for at least a qualitative information. For example, organelles within the cell with higher stiffness or density than the surrounding water increase the factors $f_{1}$ or $f_{2}$ of the overall cell. With such approaches, possibly insights into the cell's internal structure might be inferred from their acoustophoretic behavior.

\subsection{Numerical simulations on the acoustic radiation force}

In this section, the analytic calculations of hollow particles (Sect. 2.2) are compared to and validated with numerical simulations of the acoustic radiation force. The numerical simulations are computationally intensive, complex and time-consuming. However, their validation of our former analytic derivations is crucial, so in future, the expedient analytic equations can be used by a broad audience for significant and simple acoustophoretic evaluations.

The former analytic calculations are valid under the assumptions of Gor'kov's theory, namely in the longwavelength range. We are considering numerical simulations, because then Gor'kov's theory and its assumptions can be circumvented by calculating the acoustic radiation force on a particle (in an inviscid fluid) with the more fundamental equation (Bruus 2012b; Dual et al. 2012; Yosioka and Kawasima 1955; Mishra et al. 2012)

$$
\begin{aligned}
\mathbf{F}= & \frac{1}{2} \rho_{w a} \int_{S_{0}}\left[\left\langle v_{1}^{2}\right\rangle-\frac{1}{\rho_{w a}^{2} c_{w a}^{2}}\left\langle p_{1}^{2}\right\rangle\right] \mathbf{n} \mathrm{d} S \\
& -\rho_{w a} \int_{S_{0}}\left\langle\left(\mathbf{n} \cdot \mathbf{v}_{\mathbf{1}}\right) \mathbf{v}_{\mathbf{1}}\right\rangle \mathrm{d} S
\end{aligned}
$$

where the integration takes place over an arbitrary fixed surface $S_{0}$ enclosing the particle. The calculation of $\mathbf{F}$ with the above equation is not restricted to the long-wavelength range; however, it requires a particle to be placed in the simulation domain at a specific location. This requirement clarifies the value of the convenient Gor'kov approach, which allows to calculate a force field directly from the pressure- and velocity fields without any particle in the simulation domain.

We chose the software Comsol Multiphysics ${ }^{\circledR}$ to evaluate the above expression. In a three-dimensional modeling space, a single particle was placed in a specified one-dimensional standing wave field of $p_{1}(x)=$ 


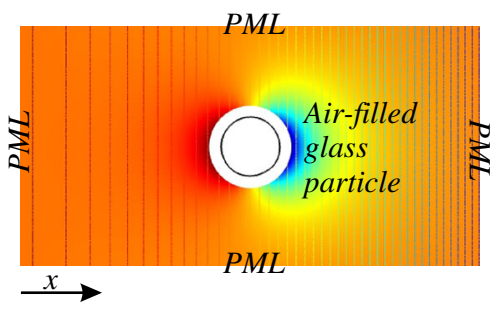

Fig. 5 Illustration of the three-dimensional acoustic numerical simulation. An air-filled particle is placed in a preset one-dimensional standing wave field, whose sinusoidal pressure field is visualized with a contour plot (vertical lines). The field plot represents the scattered pressure field, which is absorbed by perfectly matched layers (PML) at the borders of the modeling space

$p_{a} \cos \left(k_{x} x\right) \mathrm{e}^{-\mathrm{i} \omega t}$ as illustrated in Fig. 5. The particle was located in the middle between the pressure node and antinode in order to experience maximal acoustic radiation force. The simulations consisted of two acoustic domains, namely the surrounding water and an air core in the particle, as well as one linear elastic domain for the particle shell of glass. The corresponding one-dimensional analytic calculation (Bruus 2012b)

$F=\mathbf{F} \cdot \mathbf{e}_{x}=4 \pi\left(\frac{f_{1}}{3}+\frac{f_{2}}{2}\right) k_{x} r_{o}^{3} E_{a c} \sin \left(2 k_{x} x\right)$

with the acoustic energy density $E_{a c}=p_{a}^{2} /\left(4 \rho_{w a} c_{w a}^{2}\right)$ and $f_{1} f_{2}$ according to Eq. 11 is compared to the simulation results of an air-filled glass particle in the plot of Fig. 6 for a exemplary acoustic pressure amplitude of $p_{a}=0.2 \mathrm{MPa}$ (Barnkob et al. 2010). The agreement between the analytic calculation and the simulation verifies the results for the chosen frequency and radius values which lead to the parameter $k_{x} r_{o}=0.11$, which is a typical value in applied acoustophoresis.

With regard to the long-wavelength constraint, we build on numerical simulations to characterize a limit up to which the analytic calculations are valid. Figure 7 shows a plot over some magnitudes of the $k_{x} r_{o}=\omega r_{o} / c_{w a}$ value. The dimensionless quantity $Y=F /\left(S E_{a c}\right)$ [called $Y$ factor or acoustic radiation pressure function (Hasegawa et al. 1993)] is plotted, which is the force $F$ per cross-sectional area of the particle $S=\pi r_{o}^{2}$ and per energy density $E_{a c}$. For the smaller $k_{x} r_{o}$, the long-wavelength assumption $r_{o}<<\lambda$ of Gor'kov's derivation is met. Therefore, the analytic calculation matches the simulations well, and we conclude that it is a reasonable approximation up to about $k_{x} r_{o} \approx$ 0.3 , so the typical range of applied acoustophoresis is well covered and our analytical approximations hold well in this range. However, for larger $k_{x} r_{o}$, the approximation error becomes increasingly large: At $k_{x} r_{o}=0.3$, the error amounts to $7 \%$ for $\alpha=0$, to $11.5 \%$ for $\alpha=0.85$ and to $2.5 \%$ for $\alpha=0.93$. The relative error appears different for these $\alpha$ values because of differently weighted $f_{1}$ and $f_{2}$.

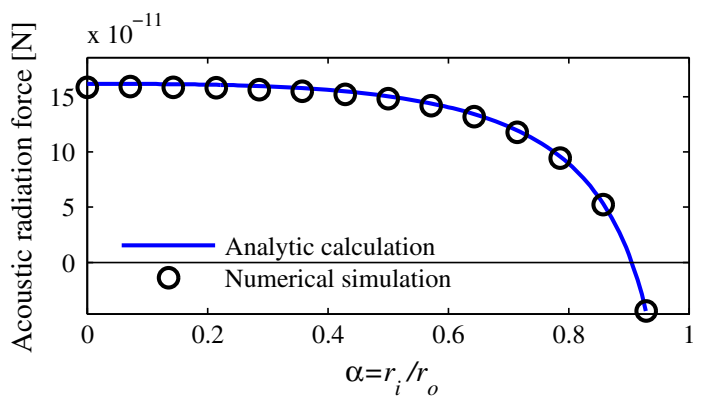

Fig. 6 Acoustic radiation force on the example of a hollow glass particle with radius $r_{o}=7 \mu \mathrm{m}$ in a one-dimensional standing wave of 3.7 MHz. The comparison between the analytic curve and the simulation results with the more general Eq. 19 shows good agreement, verifying the former in the long-wavelength range, here with $k_{x} r_{o}=0.11$

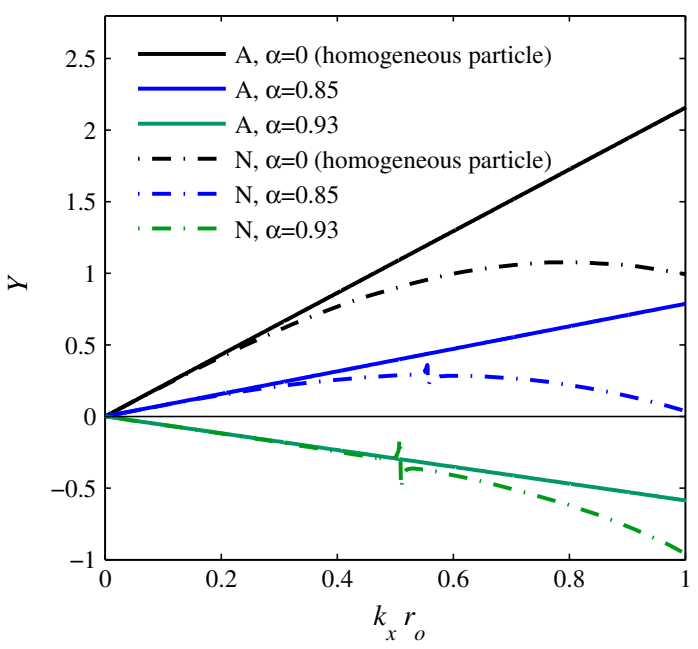

Fig. $7 Y$ factor (acoustic radiation pressure function), comparing analytic calculations "A" on homogeneous and hollow glass particles with numerical simulations "N" of homogeneous and air-filled particles for 3 exemplary $\alpha=r_{i} / r_{o}$ values. For small $k_{x} r_{o}$ values, the analytic approximation is valid and matches the simulation well. As expected and characterized with this plot, for higher $k_{x} r_{o}$ valueswhere the long-wavelength assumption is not met-the analytic approximation is no longer valid, and inner particle resonances are visible

The difference between the analytic results and the simulations at large $k_{x} r_{o}$ values is comprehensible: In the longwavelength assumption, the whole particle experiences an almost uniform pressure around itself, whereas at large $k_{x}$ $r_{o}$ the relatively large particle extends over a wide area of the wave, so the pressure on one side of the particle differs from the pressure on its other side, which affects the wave scattering and thus the radiation force. Additionally, at larger $k_{x} r_{o}$ values, resonances of the particle core or shell occur (a core resonance is visible in the plot around $k_{x} r_{o} \approx$ 0.55 ), which are also not comprised in Gor'kov's model. 
Yet, the simulation can resolve these effects, which were also analytically described by the model of Mitri (2005).

\subsection{A two-dimensional resonance mode and its effect on hollow particles}

Motivated by the experiments presented in Sect. 3, we discuss the background theory of a two-dimensional resonance mode. This resonance mode will allow to observe an acoustophoretic response which is specific for the $f_{1}$ and $f_{2}$ factors of hollow particles. It is this dependency on two different physical parameters which proves this resonance mode to be most valuable here compared to the common one-dimensional resonance modes, where a dependency on only one parameter, the acoustophoretic contrast factor $\phi$, can be observed. We follow the theory as summarized by Bruus (2012a), where the detailed derivations are given. With first-order perturbation theory for linear acoustics of inviscid fluids, the Helmholtz equation

$\nabla^{2} p_{1}=-\frac{\omega^{2}}{c_{w a}^{2}} p_{1}$

is valid for the first-order pressure field $p_{1}$, the angular frequency $\omega=2 \pi f=k \quad c_{w a}$ of its time-harmonic oscillation, the wave number $k$ and the speed of sound $c_{w a}$ in the fluid, which is water here. Since we are considering a microfluidic chamber as acoustic domain with its height dimension being much smaller than half the acoustic wavelength $\lambda / 2$, we assume a two-dimensional pressure field in the chamber along its length $l$ and width $w$. The following pressure fields with spatial coordinates $x$ and $y$ are possible solutions to the Helmholtz equation above (with the point of origin $x=y=0$ in a chamber corner):

$p_{1}(x, y)=p_{a} \cos \left(k_{x} x\right) \cos \left(k_{y} y\right) \mathrm{e}^{-\mathrm{i} \omega t}$

with a pressure amplitude $p_{a}$, the wave numbers $k_{x}=n_{x} \pi /$ $l$ and $k_{y}=n_{y} \pi / w$ and the numbers $n_{x}=0,1,2$, ..., $n_{y}=0,1,2, \ldots$ of the eigenmode, which we refer to as the " $\left(n_{x}, n_{y}\right)$ mode". As usual in the phasor approach with the time-harmonic term $\mathrm{e}^{-\mathrm{i} \omega t}$, the physically meaningful pressure is meant to be the real part $\operatorname{Re}\left(p_{1}\right)$ of the complex $p_{1}$ value. These solutions fulfill the hardwall boundary conditions, which are a valid approximation for a water-silicon boundary as present in the experiments. The oscillation frequency of the pressure field is given as

$f_{\left(n_{x}, n_{y}\right)}=\frac{c_{w a}}{2} \sqrt{\frac{n_{x}^{2}}{l^{2}}+\frac{n_{y}^{2}}{w^{2}}}$

The corresponding first-order velocity vector field $\mathbf{v}_{1}$ follows:

$$
\begin{aligned}
& \mathbf{v}_{1}(x, y)=\frac{-\mathrm{i}}{\rho_{w a} \omega} \nabla p_{1} \\
& =\frac{\mathrm{i} p_{a}}{\rho_{w a} \omega}\left(\begin{array}{l}
k_{x} \sin \left(k_{x} x\right) \cos \left(k_{y} y\right) \\
k_{y} \cos \left(k_{x} x\right) \sin \left(k_{y} y\right)
\end{array}\right) \mathrm{e}^{-\mathrm{i} \omega t}
\end{aligned}
$$

The time-averaged squared pressure and velocity fields which determine the Gor'kov potential yield

$$
\begin{aligned}
& \left\langle p_{1}^{2}\right\rangle=\frac{p_{a}^{2}}{2} \cos ^{2}\left(k_{x} x\right) \cos ^{2}\left(k_{y} y\right) \\
& \left\langle v_{1}^{2}\right\rangle=\frac{p_{a}^{2}}{2 \rho_{w a}^{2} \omega^{2}}\left\{k_{x}^{2} \sin ^{2}\left(k_{x} x\right) \cos ^{2}\left(k_{y} y\right)+k_{y}^{2} \cos ^{2}\left(k_{x} x\right) \sin ^{2}\left(k_{y} y\right)\right\}
\end{aligned}
$$

Previous experimental work in two-dimensional acoustophoresis based on the superposition of two onedimensional modes, e.g., a $(1,0)$ mode with a $(0,1)$ mode in Manneberg et al. (2008b) with different driving frequencies. Oberti et al. (2007) explained such superpositions in theory and experiments for two standing waves with the same as well as differing driving frequencies. In contrast, in our experiments, the intrinsically two-dimensional $(1,1)$ mode will be employed, because it has a different specific effect on hollow and homogeneous particles as outlined in this section. Following Eq. 23, this mode occurs at a frequency $f_{(1,1)}=\sqrt{2} f_{(1,0)}$ in square chambers.

In the $(1,1)$ mode, the $\left\langle p_{1}^{2}\right\rangle$ term has a different analytical form than the $\left\langle v_{1}^{2}\right\rangle$ term, as it becomes clear in the plot of Fig. 8a, b. Therefore, in this mode, the pressure and velocity term form a qualitatively different and unique Gor'kov potential for each particle characteristics $f_{1}$ and $f_{2}$, resulting in a unique particle accumulation pattern for each particle type. This is different to the superposition of one-dimensional resonance modes, where the $\left\langle p_{1}^{2}\right\rangle$ and $\left\langle v_{1}^{2}\right\rangle$ terms showed the same shape as plotted in Dual et al. (2012), Fig. 4, for the superposition of a $(2,0)$ and a $(0,2)$ mode. There, only two qualitatively different cases of Gor'kov potential fields were observed, namely the case with a positive or a negative acoustic contrast factor $\phi$. Beyond those two cases, the particle characteristics only influenced quantitatively the magnitude, but not qualitatively the shape of the Gor'kov potential.

Generally speaking, a positive and negative factor $f_{1}$ contributes forces toward the minima and maxima of the $\left\langle p_{1}^{2}\right\rangle$ field (pressure nodes and antinodes), respectively. A positive and negative factor $f_{2}$ contributes forces toward the maxima and minima of the $\left\langle v_{1}^{2}\right\rangle$ field (velocity antinodes and nodes), respectively. Therefore, in the $(1,1)$ mode, the achievable particle patterns can be classified in 4 categories, depending on positive and negative factors $f_{1}$ and $f_{2}$, 

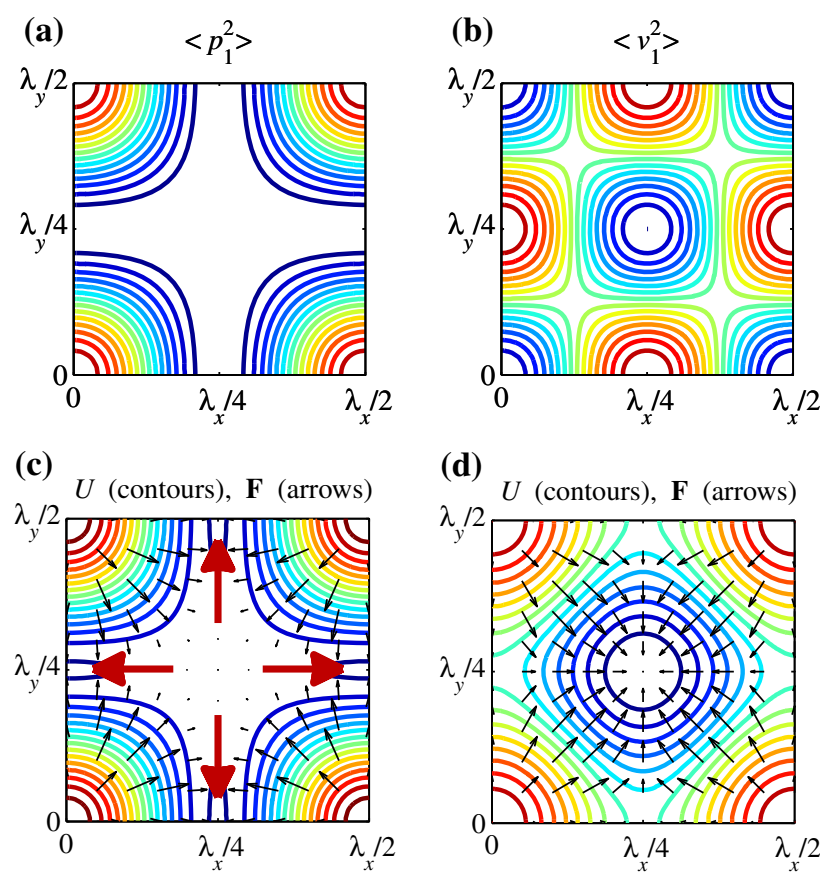

(d)

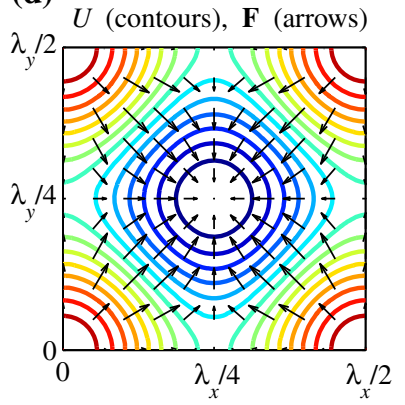

Fig. 8 Contour plots in $x$ - and $y$-direction in the $(1,1)$ mode (red maxima, blue minima, zero). The pressure field in a and the velocity field in $\mathbf{b}$ show a differently shaped function. The particle-dependent Gor'kov potential $U$ comprises both these fields; therefore, each particle type exhibits a differently shaped $U$. In c, the Gor'kov potential for typical solid particles with $f_{1}>0, f_{2}>0$ (copolymer, see Table 1) is shown. It is mostly influenced by the pressure term from $\mathbf{a}$, so it forms a cross shape. The velocity term causes the particleattracting potential minimum (blue) to be located at the 4 spots that are marked with red arrows. In d, the Gor'kov potential for hollow glass particles (listed in Table 1) with $f_{1}>0$ and $f_{2}<0$ is fundamentally different to the one in c. These hollow particles are attracted to the center $x=\lambda_{x} / 4, y=\lambda_{y} / 4$, which corresponds to the pressure and velocity minima (color figure online)

which will allow us to distinguish acoustophoretically between hollow and homogeneous particles.

Representing particles with $f_{1}>0$ and $f_{2}>0$, the Gor'kov potential for homogeneous copolymer particles is shown in Fig. 8c (material parameters in Table 1). A large $f_{1}$ and small $f_{2}$ lead to a dominating influence of the $\left\langle p_{1}^{2}\right\rangle$ term, arranging the particles in a cross shape, and a minor influence of the $\left\langle v_{1}^{2}\right\rangle$-term, which attracts the particles slightly toward the 4 spots at the chamber edges.

However, the $(1,1)$ mode has a different effect on hollow glass particles with $f_{1}>0$ and $f_{2}<0$ (here Kisker $P B G H-18$ ). As the force vectors $\mathbf{F}$ in Fig. 8d show, these hollow particles will be strongly attracted to the chamber center because there the pressure as well as the velocity term are minimal. This finding will be shown experimentally in the next section.

Notably, the $(1,1)$ mode is ideal to trap the mentioned hollow particles in a microfluidic chamber. Its Gor'kov potential looks quite similar to the one of a superposed (1, $0)$ and $(0,1)$ mode trap for homogeneous particles, yet those superposed traps cannot trap particles with $\phi<0$ or $\phi \approx 0$. In the $(1,1)$ mode, the mentioned hollow glass particles with $\phi=0.02$ experience a maximal force in the chamber which is about 10 times higher than in a trap with switching (Oberti et al. 2009) between the $(1,0)$ and the $(0,1)$ mode with the same pressure amplitude.

Likewise, third and fourth cases in the $(1,1)$ mode for particles with $f_{1}<0, f_{2}>0$ and $f_{1}<0, f_{2}<0$ (e.g., small air bubbles) can be derived. (The behavior of air bubbles depends on their size (Blake 1949). Here, we mean air bubbles which are smaller than their resonant size, for larger air bubbles further physical effects come into play.)

In conclusion, the calculation of the force fields for particles in the $(1,1)$ mode shows to be highly dependent on the particle characteristics. Conversely, when we see the particle pattern formed by a large amount of particles accumulated in the chamber, their particle characteristics can be deduced. The specific outlines of the particle patterns in the following experiments of Fig. 10 reveal their ratio between $f_{1}$ and $f_{2}$. This consideration might represent a novel possibility for particle characterization.

Particle-dependent acoustophoresis has recently gained momentum especially for experiments related to biological cells. Hartono et al. (2011) as well as Augustsson et al. (2010) report the measurement of several cell lines' contrast factors $\phi$ based on the analysis of particle trajectories in a $(1,0)$ mode. Such measurements of mechanical properties of cells are of great importance to identify cell types, cell differentiation and cell diseases. Similarly to the cited work, the presented $(1,1)$ mode here might also be employed for cell characterization by trajectory analysis, whereby a trajectory in the more complex $(1,1)$ mode reveals not only one parameter $\phi$, but even two parameters $f_{1}$ and $f_{2}$. Such acoustophoretic approaches for on-chip measurements of a cell's mechanical properties are advantageous as they are fast, direct and contactless.

Thinking one step further, the multidimensional particle dependency of the $(1,1)$ mode might even be harnessed for the advancement of acoustofluidic particle separation, which has recently been shown in the $(1,0)$ mode (Liu and Lim 2011; Augustsson et al. 2012); Kanazaki and Okada 2012). The higher $(1,1)$ mode enables more complex particle separation, since particles with higher and lower density or compressibility than the surrounding fluid are attracted to different spots in the chamber.

\section{Experiments in a microfluidic chamber}

\subsection{Device and experimental setup}

Experiments on hollow glass particles were achieved in the following microdevice. 
In order to provide an acoustic chamber, a microdevice with a main part of a $24 \mathrm{~mm} \times 8 \mathrm{~mm}$ silicon plate (wafer thickness $425 \mu \mathrm{m}$ ) was fabricated as illustrated in Fig. 9. In the silicon substrate, a $1.2 \mathrm{~mm} \times 1.2 \mathrm{~mm}$ fluidic chamber was dry etched $200 \mu \mathrm{m}$ deep inside by an inductive coupled plasma system. The chamber was covered with a glass plate (thickness $500 \mu \mathrm{m}$ ) by anodic bonding. For the actuation of the resonances, a $4 \mathrm{~mm} \times 4 \mathrm{~mm}$ piezoelectric transducer (thickness $1 \mathrm{~mm}$, Ferroperm piezoceramics Pz26) was glued on the bottom of the device with conductive epoxy (Epo-Tek H20E).

As proposed in earlier work (Oberti et al. 2007; Neild et al. 2007), the piezoelectric transducer had been prepared by cutting its back side superficially into smaller segmented electrodes. Figure $9 \mathrm{~b}$ shows the resulting strip electrodes of $800 \mu \mathrm{m}$ width, whereas electrode 1 and 2 are especially intended for excitation in $x$ - and $y$-direction, respectively.

For the experiments, the piezoelectric transducer was excited by a function generator (Stanford Research, DS345) connected to an amplifier (ENI, 2100L). The applied excitation voltages ranged between 20 and $40 V_{\text {rms. }}$. Both the electrodes 1 and 2 were connected to this voltage excitation at the same time. The advantage of this electrical wiring lies in its simplicity: Only one frequency generator and amplifier are needed, but still an excitation in two

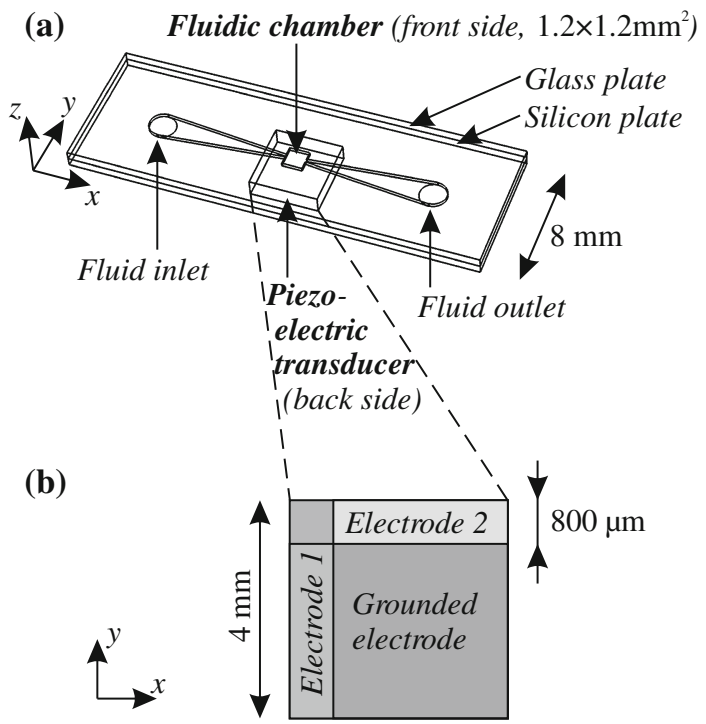

Fig. 9 a Three-dimensional sketch of the $24 \mathrm{~mm} \times 8 \mathrm{~mm}$ silicon microdevice. The centered fluidic chamber, etched in a silicon substrate, was filled through a conic inlet channel with attached tube on the device back side. The piezoelectric transducer underneath the chamber was further patterned as shown in $\mathbf{b}$, the close view on the back side electrode of the $4 \mathrm{~mm} \times 4 \mathrm{~mm} \times 1 \mathrm{~mm}$ piezoelectric transducer. The strip electrodes 1 and 2 were contacted for excitation in $x$ - and $y$-direction. The rest of the back side electrode as well as the electrode on the other side of the piezoelectric transducer were on ground potential

directions is achieved. For filling of the fluidic chamber with a liquid, a conic inlet and outlet were also etched as illustrated. At the end of the conic inlet and outlet, the silicon plate was etched through from the back side, so that two circular holes were formed. In order to connect these microscale holes to flexible macroscale tubes, two small blocks of polydimethylsiloxane (PDMS) of about $7 \mathrm{~mm} \times 7 \mathrm{~mm} \times 4 \mathrm{~mm}$ were bonded on the silicon back side, centered above the holes. Through the PDMS blocks, a hole of about $2 \mathrm{~mm}$ diameter had been punched, where flexible silicone tubes could easily be introduced by a press fit. The bonding of the PDMS blocks on the silicon device was enabled by oxygen plasma activation of the surfaces, which is a widely reported method (Duffy et al. 1998).

The inlet and outlet are designed quite small at the chamber entrance because of concerns that they might influence the acoustic field within the chamber. On our design, these concerns were allayed with confirmative experiments in the $(0,1)$ and $(1,0)$ mode as well as twodimensional acoustic eigenmode simulations of the water domain. They showed that the proposed pressure field of

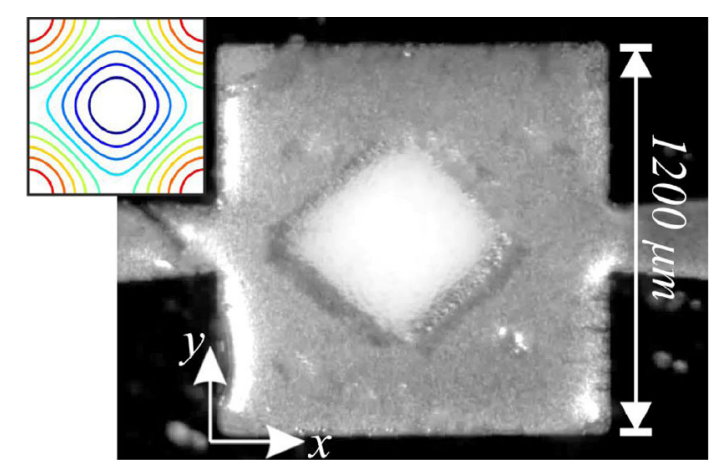

(a) Hollow glass particles (Kisker $P B G H-18$ ) $\varnothing 13.9 \mu \mathrm{m}$, centered square, $870 \mathrm{kHz}$

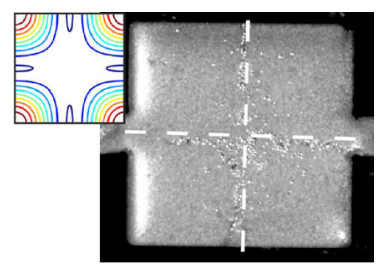

(b) Copolymer particles $\varnothing 17 \mu \mathrm{m}, \quad$ cross shape, $880 \mathrm{kHz}$

Fig. 10 Experimental results for the $(1,1)$ mode within a water-filled microfluidic chamber in a device according to Fig. 9. The key experiment in a with hollow glass particles of $f_{1}>0, f_{2}<0$ coincides well to the Gor'kov potential $U$ in the small inserted plot top left (Fig. 8d). The cross shape in $\mathbf{b}$ is mostly based on the pressure field, whereas the velocity field additionally draws the (translucent) particles toward the 4 marked spots at the chamber border in c. Again these results correspond well to the Gor'kov potential minima in the plots top left (see also Fig. 8c). (The light, vertical spots along the left chamber walls are light reflections) 
Eq. 22 is not significantly influenced by the inlet and outlet. Their conic shape was chosen to prevent clogging of particles.

The described chamber can easily be scaled down, both in terms of fabrication technology and physical working principle. The resonance frequencies correlate inversely with the chamber side length according to Eq. 23, so the chamber can be scaled down more than 10 times without exceeding the typical acoustophoretic frequency range of $<10 \mathrm{MHz}$ (Wiklund 2012) for the experiments in the next section.

\subsection{Experimental results}

The analytical discussions (Sects. 2.2 and 2.5) are experimentally verified in this section on the example of 3 different particle types.

The key experiment was conducted with hollow glass particles, Kisker $P B G H-18$. Their image was taken with a laser scanning microscope (Zeiss LSM 5 Pascal) in Fig. 1, where the focal plane crosses the sphere center. These particles have a specified outer diameter of $18 \mu \mathrm{m}$; however, in a measurement on 50 particles, an average diameter of $2 r_{o}=13.9 \mu \mathrm{m}$ with a standard deviation of $4.2 \mu \mathrm{m}$ was found. The base material of the hollow particles is borosilicate, whose precise material parameters are not known. Instead, we calculated with the material parameters of the well-defined Pyrex glass (Bruus 2012b), which is also a borosilicate glass. The averaged density $\bar{\rho}_{p}$, the bulk material density $\rho$ and $r_{o}$ given, $r_{i}$ was calculated with agreement to the LSM results. The calculation of the tabulated $f_{1}>0$ and $f_{2}<0$ follows Eq. 11 . All the particle parameters are listed in Table 1.

By comparing the experimental result of the $(1,1)$ mode in Fig. 10a with its Gor'kov potential in Fig. 8d, a match between theory and experiments is found. The particles could be aligned quickly, precisely and reproducibly to the shown square-edged particle pattern in the chamber center, revealing high acoustic radiation forces. In the theoretical section, the influence of secondary acoustic forces (Laurell et al. 2007) (particle-particle interactions, e.g., Bjerknes forces) is neglected; nevertheless, it might be subject of future work.

For the category of typical homogeneous, solid particles with $f_{1}>0$ and $f_{2}>0$, copolymer and Ca-alginate particles were considered. For the copolymer particles, the result in Fig. 10b was achieved in the $(1,1)$ mode: The particles accumulated in a cross-like shape, representing the $\left\langle p_{1}^{2}\right\rangle$ field. Because of $f_{2}<<f_{1}$ for copolymer, the influence of the $\left\langle v_{1}^{2}\right\rangle$ field was found to be too weak to attract the particles to the edges as expected from Fig. 8c. As observed in the result of Fig. 10c, the larger Ca-alginate particles were clearly attracted to the minima of $\left\langle p_{1}^{2}\right\rangle$ and the maxima of $\left\langle v_{1}^{2}\right\rangle$ as plotted in Fig. 8a, b. The influence of the velocity field was found to be higher than for the copolymer particles, because $f_{2}$ is relatively large compared to $f_{1}$ for Ca-alginate.

The calculated resonance frequency according to Eq. 23 for the chamber in Fig. 10a, b with $l=w=1.2 \mathrm{~mm}$ is $f_{(1,1)}=882 \mathrm{kHz}$, the one for the chamber in Fig. 10c with $l=1.4 \mathrm{~mm}, \quad w=1.2 \mathrm{~mm}$ is $f_{(1,1)}=822 \mathrm{kHz}$, which compares well to the corresponding experimentally tested values of 880,870 and $809 \mathrm{kHz}$ in these figures, respectively. The small frequency deviations are believed to be caused by compliant boundaries, temperature shifts (Augustsson et al. 2011), manufacturing and electrical wiring imperfections, imprecise material parameters, interfering resonances of the piezoelectric element and clamping influences. Regarding manufacturing imperfections, in particular the dry etching process cannot provide perfectly vertical chamber walls. Therefore, the length and width of the chamber at its top and bottom differ, which influences the resonance frequency band.

Acoustophoretic systems offer great potential for the trapping of microparticles, as reviewed by Evander and Nilsson (2012). Acoustic trapping enables e.g., enhanced particle-based bioassays, facilitated interaction studies of both cells and particles, enrichment of low concentration samples and particle washing or fractioning. More specific, Evander et al. (2007) and Hultström et al. (2007) reported particle traps where cells are held against a continuous flow of cell culture medium. In order to address this biotechnological scope with our findings, an experiment with exchange of trapped particles' suspending fluid is reported in Fig. 11. A video in the online resources further documents this experiment. At the beginning of the experiment in Fig. 11a, the centered cluster of trapped particles is circular, whereas the larger cluster in Fig. $11 \mathrm{~b}$ shows a square form, which is consistent with the form of the potential lines in Fig. 8d. In order to visualize a fluid exchange, then a blue dye was introduced to the liquid flow. Whereas in Fig. 11b, the inflow of the dye can be seen; about $35 \mathrm{~s}$ later in Fig. 11c, the fluid in the whole chamber was dyed. This trapping under a constant flow of the suspending liquid allows for the control of the outer conditions of the particles or for a downstream analysis of compounds released by biologically or chemically relevant particle materials such as, e.g., coated hollow particles with bounded analytes or cells and agglutination assays (Wiklund et al. 2013). Our microfluidic device is also interesting for various biological approaches where long-term observation of trapped particles is required. The trapping efficiency was found to be dependent on the size of the aggregated particle cluster. After the cluster has grown to the size shown in the video, incoming particles were found 

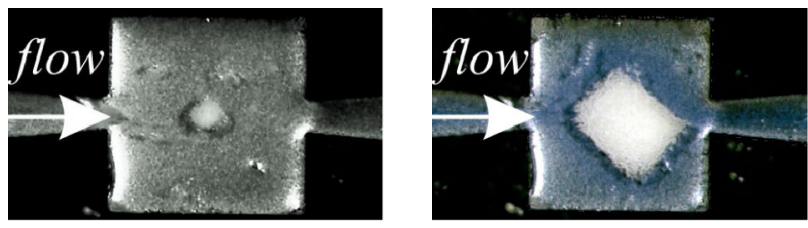

(a) Growing cluster of (b) Beginning of the inflow trapped particles of a blue dye

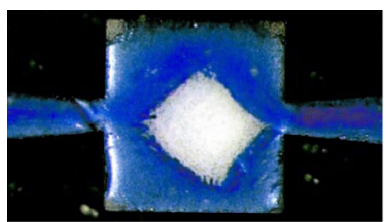

(c) Medium exchanged

Fig. 11 Hollow particles caught in the microfluidic chamber center by the acoustophoretic trap of the $(1,1)$ mode. After a cluster had been formed from $\mathbf{a}$ to $\mathbf{b}$ with a flow of $20 \mu \mathrm{l} / \mathrm{min}$ from the inlet on the left side, we were able to exchange the suspending water for a blue dye from $\mathbf{b}$ to $\mathbf{c}$ while monitoring the particles. (The image colors were edited for better visibility, a raw video is provided in the online resources.) (color figure online)

to expel trapped particles; otherwise, the trapped particles were observed to be stable. The experiments were outlined in the $x$ - and $y$-direction of an in-plane chamber; nevertheless, they might also be of relevance for the twodimensional focusing of hollow glass particles in a microfluidic channel (Manneberg et al. 2008a) in $z$ - and $x$ or $y$ - direction.

\section{Conclusions}

The study at hand aimed at the advancement of acoustophoresis toward hollow particles and particles with liquid or solid cores and shells.

Hollow and core-shell particles showed to be acoustophoretically interesting since they allow a designed, tunable and functionalized particle behavior, especially regarding the strongly varying density-dependent influence of the velocity field which is determined by the factor $f_{2}$. In particular, hollow solid particles with a negative acoustic contrast factor $\phi<0$ are promising in light of their biotechnological applications. Such "negative acoustic contrast particles" have recently received increased attention for biotechnological applications: Cushing et al. (2013) demonstrated the separation of red blood cells with $\phi>0$ from functionalized negative acoustic contrast particles with $\phi<0$ for the rapid detection of low concentrations of biomarkers.

Similarly, hollow particles with $\phi<0$ might be separated acoustophoretically from particles with $\phi>0$ as described by Laurell et al. (2007).

As a further application example of hollow particles, in a cell suspension, cells of a first type become separable from cells of a second type by chemically binding the first to a carrier particle with $\phi>0$ and binding the latter to a hollow carrier particle with $\phi<0$. Afterward, these binded complexes can be separated acoustophoretically as outlined above. The same procedure is feasible for a wide range of biological sample types, which enables affinity-specific extraction and sample decomplexing (Augustsson and Laurell 2012). Another promising application of hollow particles in acoustophoresis is based on the fact that their buoyancy can be adjusted. By designing hollow particles with the same density as their suspending fluid with $f_{2}=0$, the frequent problem of particle sedimentation on the microfluidic bottom might be solved.

In the course of experimental evaluations, we observed the two-dimensional $(1,1)$ resonance mode to be highly dependent on the mechanical particle characteristics. Its particle accumulation pattern changes gradually with the particle parameters between 4 different cases $\left(f_{1}, f_{2}>\right.$ I $<0$ ), unlike one-dimensional acoustophoresis, where only 2 cases occur $(\phi>/<0)$. As outlined in the paper, this particle-dependent acoustophoretic behavior offers potential which might be harnessed for particle characterization as well as particle separation.

Acknowledgments The authors would like to express their gratitude for funding by ETH Zurich and the Swiss National Science Foundation, SNF No. 200021_126986.

\section{References}

Augustsson P, Laurell T (2012) Acoustofluidics 11: affinity specific extraction and sample decomplexing using continuous flow acoustophoresis. Lab Chip 12:1742-1752. doi:10.1039/C2LC40200A

Augustsson P, Barnkob R, Grenvall C, Deierborg T, Brundin P, Bruus $\mathrm{H}$, Laurell $\mathrm{T}$ (2010) Measuring the acoustophoretic contrast factor of living cells in microchannels. In: Proceedings of 14th MicroTAS 14

Augustsson P, Barnkob R, Wereley ST, Bruus H, Laurell T (2011) Automated and temperature-controlled micro-piv measurements enabling long-term-stable microchannel acoustophoresis characterization. Lab Chip 11:4152-4164. doi:10.1039/C1LC20637K

Augustsson P, Magnusson C, Nordin M, Lilja H, Laurell T (2012) Microfluidic, label-free enrichment of prostate cancer cells in blood based on acoustophoresis. Anal Chem 84(18):7954-7962. doi:10.1021/ac301723s

Barnkob R, Augustsson P, Laurell T, Bruus H (2010) Measuring the local pressure amplitude in microchannel acoustophoresis. Lab Chip 10:563-570. doi:10.1039/B920376A

Blake FG (1949) Bjerknes forces in stationary sound fields. J Acoust Soc Am 21(5):551-551. doi:10.1121/1.1906547

Bower A (2009) Applied mechanics of solids. CRC Press, Boca Raton, FL

Bruus H (2012a) Acoustofluidics 2: perturbation theory and ultrasound resonance modes. Lab Chip 12:20-28. doi:10.1039/ C1LC20770A

Bruus $H$ (2012b) Acoustofluidics 7: the acoustic radiation force on small particles. Lab Chip 12:1014-1021. doi:10.1039/C2LC 21068A 
Bruus H, Dual J, Hawkes J, Hill M, Laurell T, Nilsson J, Radel S, Sadhal S, Wiklund M (2011) Forthcoming lab on a chip tutorial series on acoustofluidics: acoustofluidics-exploiting ultrasonic standing wave forces and acoustic streaming in microfluidic systems for cell and particle manipulation. Lab Chip 11:3579-3580. doi:10.1039/C1LC90058G

Caille N, Thoumine O, Tardy Y, Meister JJ (2002) Contribution of the nucleus to the mechanical properties of endothelial cells. J Biomech 35(2):177-187. doi:10.1016/S0021-9290(01)00201-9

Choi SW, Zhang Y, Xia Y (2009) Fabrication of microbeads with a controllable hollow interior and porous wall using a capillary fluidic device. Adv Funct Mater 19(18):2943-2949. doi:10.1002/ adfm.200900763

Cushing KW, Piyasena ME, Carroll NJ, Maestas GC, López BA, Edwards BS, Graves SW, López GP (2013) Elastomeric negative acoustic contrast particles for affinity capture assays. Anal Chem 85(4):2208-2215

Dual J, Hahn P, Leibacher I, Moller D, Schwarz T, Wang J (2012) Acoustofluidics 19: ultrasonic microrobotics in cavities: devices and numerical simulation. Lab Chip 12:4010-4021. doi:10.1039/ C2LC40733G

Duffy DC, McDonald JC, Schueller OJA, Whitesides GM (1998) Rapid prototyping of microfluidic systems in poly(dimethylsiloxane). Anal Chem 70(23):4974-4984. doi:10.1021/ac980656z

Evander M, Nilsson J (2012) Acoustofluidics 20: applications in acoustic trapping. Lab Chip 12:4667-4676. doi:10.1039/ C2LC40999B

Evander M, Johansson L, Lilliehorn T, Piskur J, Lindvall M, Johansson S, Almqvist M, Laurell T, Nilsson J (2007) Noninvasive acoustic cell trapping in a microfluidic perfusion system for online bioassays. Anal Chem 79(7):2984-2991. doi:10.1021/ ac061576v

Gor'kov LP (1962) On the forces acting on a small particle in an acoustical field in an ideal fluid. Soviet Phys Doklady 6(9):773-775

Griffiths AD, Tawfik DS (2006) Miniaturising the laboratory in emulsion droplets. Trend Biotechnol 24(9):395-402. doi:10. 1016/j.tibtech.2006.06.009

Hartono D, Liu Y, Tan PL, Then XYS, Yung LYL, Lim KM (2011) On-chip measurements of cell compressibility via acoustic radiation. Lab Chip 11:4072-4080. doi:10.1039/C1LC20687G

Hasegawa T, Hino Y, Annou A, Noda H, Kato M, Inoue N (1993) Acoustic radiation pressure acting on spherical and cylindrical shells. J Acoust Soc Am 93(1):154-161. doi:10.1121/1.405653

Hennequin Y, Pannacci N, de Torres CP, Tetradis-Meris G, Chapuliot S, Bouchaud E, Tabeling P (2009) Synthesizing microcapsules with controlled geometrical and mechanical properties with microfluidic double emulsion technology. Langmuir 25(14): 7857-7861. doi:10.1021/la9004449, pMID: 19594177

Hultström J, Manneberg O, Dopf K, Hertz H, Brismar H, Wiklund M (2007) Proliferation and viability of adherent cells manipulated by standing-wave ultrasound in a microfluidic chip. Ultrasound Med Biol 33(1):145-151. doi:10.1016/j.ultrasmedbio.2006.07. 024

Jeong WC, Choi MK, Lim CH, Yang SM (2012) Microfluidic synthesis of atto-liter scale double emulsions toward ultrafine hollow silica spheres with hierarchical pore networks. Lab Chip 12:5262-5271. doi:10.1039/C2LC40886D

Kanazaki T, Okada T (2012) Two-dimensional particle separation in coupled acoustic-gravity-flow field vertically by composition and laterally by size. Anal Chem 84(24):10750-10755. doi:10. $1021 /$ ac302637e

Laurell T, Petersson F, Nilsson A (2007) Chip integrated strategies for acoustic separation and manipulation of cells and particles. Chem Soc Rev 36:492-506. doi:10.1039/B601326K
Lilliehorn T, Nilsson M, Simu U, Johansson S, Almqvist M, Nilsson J, Laurell T (2005) Dynamic arraying of microbeads for bioassays in microfluidic channels. Sensors Actuators B 106:851-858

Lim C, Zhou E, Quek S (2006) Mechanical models for living cells-a review. J Biomech 39(2):195-216. doi:10.1016/j.jbiomech.2004. 12.008

Liu Y, Lim KM (2011) Particle separation in microfluidics using a switching ultrasonic field. Lab Chip 11:3167-3173. doi:10.1039/ C1LC20481E

Manneberg O, Svennebring J, Hertz HM, Wiklund M (2008a) Wedge transducer design for two-dimensional ultrasonic manipulation in a microfluidic chip. J Micromech Microeng 18(9):095025

Manneberg O, Vanherberghen B, Svennebring J, Hertz HM, Önfelt B, Wiklund M (2008b) A three-dimensional ultrasonic cage for characterization of individual cells. Appl Phys Lett 93(6): 063901. doi:10.1063/1.2971030

Manneberg O, Vanherberghen B, Onfelt B, Wiklund M (2009) Flowfree transport of cells in microchannels by frequency-modulated ultrasound. Lab Chip 9:833-837. doi:10.1039/B816675G

Mishra P, Glynne-Jones P, Boltryk RJ, Hill M (2012) Efficient finite element modeling of acoustic radiation forces on inhomogeneous elastic particles. AIP Conf Proc 1433(1):753-756. doi:10.1063/ 1.3703290

Mitri F (2005) Acoustic radiation force acting on elastic and viscoelastic spherical shells placed in a plane standing wave field. Ultrasonics 43(8):681-691. doi:10.1016/j.ultras.2005.03.002

Neild A, Oberti S, Dual J (2007) Design, modeling and characterization of microfluidic devices for ultrasonic manipulation. Sensors Actuators B Chem 121(2):452-461. doi:10.1016/j.snb. 2006.04.065

Oberti S, Neild A, Dual J (2007) Manipulation of micrometer sized particles within a micromachined fluidic device to form twodimensional patterns using ultrasound. J Acoust Soc Am 121(2):778-785. doi:10.1121/1.2404920

Oberti S, Neild A, Quach R, Dual J (2009) The use of acoustic radiation forces to position particles within fluid droplets. Ultrasonics 49(1):47-52. doi:10.1016/j.ultras.2008.05.002

Salsac AV, Zhang L, Gherbezza JM (2011) Measurement of the mechanical properties of alginate beads using ultrasounds. In: 19ème Congrès Français de Mécanique. doi:http://hdl.handle. net/2042/36663

Svennebring J, Manneberg O, Skafte-Pedersen P, Bruus H, Wiklund M (2009) Selective bioparticle retention and characterization in a chip-integrated confocal ultrasonic cavity. Biotechnol Bioeng 103(2):323-328. doi:10.1002/bit.22255

Utada AS, Lorenceau E, Link DR, Kaplan PD, Stone HA, Weitz DA (2005) Monodisperse double emulsions generated from a microcapillary device. Science 308(5721):537-541. doi:10. 1126/science. 1109164

Vanherberghen B, Manneberg O, Christakou A, Frisk T, Ohlin M, Hertz HM, Onfelt B, Wiklund M (2010) Ultrasound-controlled cell aggregation in a multi-well chip. Lab Chip 10:2727-2732. doi:10.1039/C004707D

Wiklund M (2012) Acoustofluidics 12: biocompatibility and cell viability in microfluidic acoustic resonators. Lab Chip 12:2018-2028. doi:10.1039/C2LC40201G

Wiklund M, Hertz HM (2006) Ultrasonic enhancement of bead-based bioaffinity assays. Lab Chip 6:1279-1292. doi:10.1039/B609184A

Wiklund M, Radel S, Hawkes JJ (2012) Acoustofluidics 21: ultrasound-enhanced immunoassays and particle sensors. Lab Chip 12:4667-4676. doi:10.1039/C2LC41073G

Wiklund M, Radel S, Hawkes JJ (2013) Acoustofluidics 21: ultrasound-enhanced immunoassays and particle sensors. Lab Chip 13:25-39. doi:10.1039/C2LC41073G

Yosioka K, Kawasima Y (1955) Acustica 5:167-173 